\title{
Deficient pregnenolone synthesis associated with congenital adrenal hyperplasia and organelle dysfunction
}

\author{
Himangshu S Bose1,2, Alan M Rice1,3, Brendan Marshall4, Fadi Gebrail1,5, David Kupshik ${ }^{1}$ \\ and Elizabeth W Perry 4
}

'Laboratory of Biochemistry, Biomedical Sciences, Mercer University School of Medicine, Savannah, Georgia, USA, 2Memorial University Medical Center, Savannah, Georgia, USA, 3Pediatric Endocrinology and Diabetes Center, Kalispell Regional Medical Center, Kalispell, Montana, USA, ${ }^{4}$ Anatomy and Pathology, Augusta State University, Augusta, Georgia, USA, and 5Laboratory of Pathology, Memorial University Medical Center, Savannah, Georgia, USA
Correspondence should be addressed to H S Bose

Email

bose_hs@mercer.edu

\section{Summary}

Steroid hormones are essential for the survival of all mammals. In adrenal glands and gonads, cytochrome P450 side chain cleavage enzyme (SCC or CYP11A1), catalyzes conversion of cholesterol to pregnenolone. We studied a patient with ambiguous genitalia by the absence of Müllerian ducts and the presence of an incompletely formed vagina, who had extremely high adrenocorticotropic hormone (ACTH) and reduced pregnenolone levels with enlarged adrenal glands. The testes revealed seminiferous tubules, stroma, rete testis with interstitial fibrosis and reduced number of germ cells. Electron microscopy showed that the patient's testicular mitochondrial size was small with little SCC expression within the mitochondria. The mitochondria were not close to the mitochondria-associated ER membrane (MAM), and cells were filled with the microfilaments. Our result revealed that absence of pregnenolone is associated with organelle stress, leading to altered protein organization that likely created steric hindrance in testicular cells.

\section{Learning points:}

- Testes revealed seminiferous tubules, stroma, rete testis with interstitial fibrosis and reduced number of germ cells;

- Testicular mitochondrial size was small with little SCC expression within the mitochondria;

- Absence of pregnenolone is associated with organelle stress.

\section{Background}

Prenatal stress affects many aspects of reproduction, including sexual differentiation of the brain, development of the reproductive organs, puberty onset, reproductive behavior, gonad function and concentrations of reproductive hormones (1). In addition, the physiological consequences of stressors in a pregnant female may be transmitted to her developing fetuses, including alterations in the opioid system, as well as causing epigenetic changes to placental gene expression and alterations in the maternal hypothalamic-pituitary-adrenal (HPA) axis.
The stress-induced alterations in the maternal HPA axis, also elevate maternal glucocorticoid levels, potentially increasing the amount of glucocorticoids crossing the placenta from the mother to the fetus, and thereby increasing fetal exposure to high glucocorticoids (1).

The acute response to danger consists of a relatively stereotyped series of physiological and behavioral programs that promote survival and is mediated by the HPA axis. In acute physiological stress, glucocorticoid synthesis is initiated by the hypothalamic neuropeptide, 
corticotropin-releasing hormone (CRH), which stimulates pituitary release of adrenocorticotropin hormone (ACTH). Activation of ACTH receptors in the adrenal cortex promotes glucocorticoid synthesis and secretion; glucocorticoids then act on a wide range of target tissues (1). Steroidogenic cells store very little steroid hormone; therefore, a rapid steroidogenic response requires synthesis of new steroid $(2,3,4)$. In response to ACTH, cAMP stimulates the secretion of StAR almost immediately and folded by GRP78 (5). Next, it is loaded onto the outer mitochondrial membrane (OMM) for cholesterol transport to inner mitochondrial membrane, which is then catalyzed by the cholesterol side chain cleavage enzyme, CYP11A1 or P450scc (SCC), to form the first steroid in the tissue, pregnenolone. Thus, pregnenolone is the first substrate for all steroids synthesized in the adrenals and ovary/gonads in a very complex pathway involving many genes. Any mutation in the genes involved in the pathway generally leads to diminished production of cortisol and adrenal enlargement and termed congenital adrenal hyperplasia (CAH). As a result, CAH patients usually develop salt-losing crisis with an ambiguous genitalia. In some case, physiological and laboratory data have identified patients with biochemical evidence of CAH with no known mutations in any gene participating in the steroid synthesis pathway (6).

As described earlier, elimination of SCC activity is incompatible with full-term gestation because the placenta, a fetal tissue, must produce progesterone from the second trimester for successful gestation (1). There are several SCC mutations described in the literature, including frameshift mutations, amino acid substitution, point mutations and heteroxygote formations (7). Confirmation that these mutations cause $\mathrm{CAH}$ was verified by recreating them in a pCMV vector containing SCC cDNA, followed by overexpression in nonsteroidogenic COS-1 or knockdown steroidogenic cells (8). To our surprise, analysis of protein expression by the mutants using a SCC antibody showed that their levels were identical to that of the WT protein, suggesting that the WT and mutants were processed into the mitochondria in a similar fashion $(7,8,9)$.

Reproductive organ development in mammalian systems, especially in humans, is unique for the testis and ovary, as both organs start developing from an initially bipotential tissue - the genital ridge initiated via expression of the sex-determining region of the Y-chromosome (SRY) gene (10). After ovulation, luteinizing hormone (LH) stimulates granulosa cell production of progesterone, which regulates follicular rupture and luteinization and plays critical roles in maintaining a successful pregnancy at the early embryonic stage. Therefore, the precise regulation of progesterone production in granulosa cells is necessary for maintaining reproductive function. In male fetuses, Sertoli cells are required for regulating all subsequent events in testicular development. Interstitial fetal Leydig cell production of androgens and insulin-like factors masculinize the embryo and promote testicular development (1).

We described a thorough analysis of a 46XY infant who had developed CAH due to a SCC gene mutation (7); this patient also had ambiguous genitalia and complete closure of the urogenital track. The patient's family decided to raise the baby as a female, and both testes were surgically removed, one of which was mostly degraded. We have performed a detailed analysis of the ultrastructure of the testis of the patient, analyzing the mitochondria and endoplasmic reticulum (ER) structures using light microscopy, electron microscopy (EM) and biochemical experiments since the adrenal glands and gonads are primary steroid-producing organs in which changes in the environment can affect organelle structure. We observed earlier that environmental stress on the ER affected mitochondrial metabolic activity (11), suggesting that these environmental changes can completely alter activity when only minimal changes in activity were detected following protein mutation in non-environmentally stressed conditions. During endogenous and exogenous stress, the HPA axis was activated and included CRH-stimulated ACTH release, affecting organelle architecture and resulting in the disruption of steroid synthesis (Fig. 1A). We concluded that the increased cortisol was not only due to the import and residency of mitochondrial-translocating proteins and steroidogenic enzymes, but was also mediated by the total network, which drives metabolic activity.

\section{Case presentation}

In brief, the patient was born premature with appropriate weight, length and head circumference for their gestational age. Toward the end of the first week of life, pre-onset of glucocorticoid and mineralocorticoid therapy, ACTH and plasma renin activity (PRA) levels were elevated, progesterone, 17-hydroxyprogesterone, dihydroepiandrosterone and androstenedione levels were below the detectable range of the assays used, and the sodium was mildly low, while the potassium was slightly elevated. The chromosome analysis result 
A

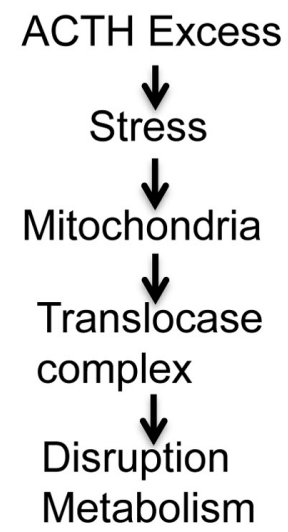

C

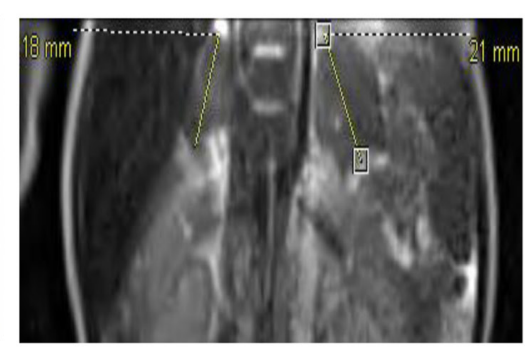

D

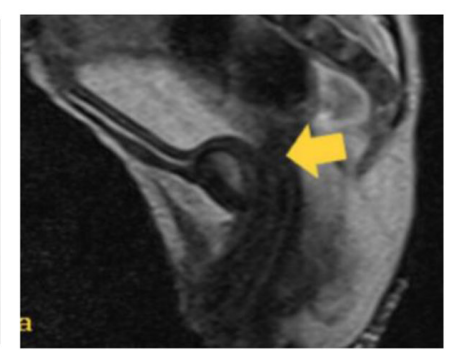

\section{Figure 1}

(A) Phenotypic changes resulting from excess ACTH. (B) Patient's picture showing the excess release of cortisol resulted pigmentation. (C) MRI showing an enlarged adrenal gland, where the right adrenal gland is larger than the left. (D) MRI showing completely blunt end of urogenital track (arrow).

was 46,XY. Magnetic resonance imaging (MRI) analysis revealed enlarged adrenal glands and an abrupt ending of a vagina with no Müllerian remnants identified. After bilateral orchiectomies at the age of approximately 1 year, a thorough histological and immunoelectron microscopic analysis of the gonads was performed.

\section{Electron microscopy}

EM was used to identify the localization of SCC in the mitochondria of testicular cells following our previously described procedure (12). Tissues were fixed in $4 \%$ paraformaldehyde and $0.2 \%$ glutaraldehyde in $0.1 \mathrm{M}$ sodium cacodylate buffer, $\mathrm{pH} 7.4$ and then dehydrated through a graded ethanol series prior to embedding in LR white resin. Sections of $75 \mathrm{~nm}$ were cut using a Leica EM UC6 ultramicrotome (Leica Microsystems) and collected on 200 mesh nickel grids. For immunogold labeling experiments, grids were blocked in $0.1 \%$ BSA in PBS (phosphate buffer saline) for $4 \mathrm{~h}$ at room temperature in a humidified atmosphere followed by incubation with a 1:1000 dilution of anti-Tim50 antibody (source), a 1:1000. dilution of anti-SCC antibody (source), a 1:100 dilution of anti-Tim23 antibody (source) and a 1:100 dilution of anti-StAR antibody (source) in $0.1 \%$ BSA overnight at $4^{\circ} \mathrm{C}$. Grids were then washed five times with PBS and floated on drops of secondary antibody conjugated to ultra-small $(<1.0 \mathrm{~nm})$ Nanogold $^{\mathrm{TM}}$ reagent (Nanoprobes, Yaphank, NY, USA) diluted 1:2000 in 0.1\% BSA in PBS for 2-4h at room temperature. Grids were washed five times with $\mathrm{PBS}$ and deionized $\mathrm{H}_{2} \mathrm{O}$ respectively, and nanogold was enhanced with HQ Silver ${ }^{\mathrm{TM}}$ (Nanoprobes) for $8 \mathrm{~min}$ followed by washing in deionized $\mathrm{H}_{2} \mathrm{O}$.

\section{Histological analysis}

Testes were fixed in zinc formalin, processed and embedded in paraffin for routine light microscopy. Sections of $5-10 \mu \mathrm{m}$ were obtained from each specimen, floated onto glass slides, stained with hematoxylin and eosin (H\&E) and examined by microscopy (11).

\section{Figure preparation and data analysis}

Images were obtained from the autoradiogram or scanning through a phosphorimager, and the data analysis was performed using Kaleidagraph or Microsoft Excel. The figures were prepared with no computer manipulation.

\section{Investigation}

\section{Study participants}

Informed consent was obtained from each participating family member as well as the parents of the patient with putative $\mathrm{CAH}$.

\section{Patient analysis}

Adrenals and gonads are the main sites of steroid synthesis. Overproduction and underproduction of mineralocorticoids, glucocorticoids and sex steroid precursors by the adrenal cortex, and sex steroids by the gonads can cause many health problems, including dangerously abnormal electrolyte and glucose imbalances and blood pressure variations, as well as virilization of $46, \mathrm{XX}$ individuals with ovaries, and undervirilization of $46, \mathrm{XY}$ individuals with testes, sometimes to the point of 
causing ambiguous genitalia (Table 1). The infant, whose testes were analyzed, had darker skin pigmentation than expected based upon parental skin pigmentations. MRI also showed enlarged adrenals (left adrenal, $23 \times 18 \mathrm{~mm}$ with a limb width of $5.4 \mathrm{~mm}$; right adrenal $20 \times 11 \mathrm{~mm}$ with limb width of $5.2 \mathrm{~mm}$; an adrenal length at or above $20 \mathrm{~mm}$ and limb width at or above $4 \mathrm{~mm}$ during the neonatal period have been reported to be enlarged and consistent with CAH; Fig. 1B). In general, the size of the adrenal is similar to the size of the kidney at birth (13), but the relative weight of the adrenal gland during weeks of $8-23$ is stable, contributing to approximately $0.4 \%$ of the total body weight. The enlarged adrenals were similar to that observed in lipoid CAH patients (8). MRI of the pelvis also revealed the presence of an incompletely formed vagina, and there was no evidence of Müllerian ducts (Fig. 1C).

Further physiological analysis of the patient showed that the testes were smaller compared to other infants his age. Since there was no scrotum or pouch-like formation with a pendulum typical of that age, the testes were surgically removed. The histology of the testes showed seminiferous tubules, stroma and rete testis (Fig. 2A). An enlarged version of Fig. $2 \mathrm{~A}$ is shown in Fig. $2 \mathrm{~B}$ and $\mathrm{C}$. Figure $2 \mathrm{~B}$ showed early interstitial fibrosis in the patient, which is not normal as compared to the unaffected tissue at that age. Although the testes had normal looking germ cells, the number of cells was reduced with small tubular size. A close look of the tissue also showed atrophy of the rete ridges and epididymis (Fig. 2C).

EM analysis showed that the mitochondria were extremely small (Fig. 2D), and OMM and inner mitochondrial membrane (OMM and IMM, respectively) were not separated even in high-power images $(0.5 \mu \mathrm{m}$; $2 \mathrm{E}-2 \mathrm{G})$.

Table 1 Clinical data of the patient on the 6th day of life (pre-treatment with glucocorticoid and mineralocorticoid medications).

\begin{tabular}{|c|c|c|}
\hline Blood test & Reference ranges & Results \\
\hline Sodium & 134-144 mmol/L & $130 \mathrm{mmol} / \mathrm{L}$ \\
\hline Potassium & $3.5-5.2 \mathrm{mmol} / \mathrm{L}$ & $5.7 \mathrm{mmol} / \mathrm{L}$ \\
\hline Glucose & $60-140$ mg/dL & $58 \mathrm{mg} / \mathrm{dL}$ \\
\hline Cortisol & & $<0.4 \mu g / d L$ \\
\hline ACTH-ICMA & $5-46 \mathrm{pg} / \mathrm{mL}$ & 3975 pg/mL \\
\hline PRA & 2-35 ng/mL/HR & $141.6 \mathrm{ng} / \mathrm{mL} / \mathrm{HR}$ \\
\hline Progesterone & & $<0.1 \mathrm{ng} / \mathrm{mL}$ \\
\hline 17-OH progesterone & $26-568 \mathrm{ng} / \mathrm{dL}$ & $<10 \mathrm{ng} / \mathrm{dL}$ \\
\hline Dihydroepiandrosterone & $<40 \mathrm{ng} / \mathrm{mL}$ & $<0.05 \mathrm{ng} / \mathrm{mL}$ \\
\hline Androstenedione & $0.8-4.46 \mathrm{ng} / \mathrm{mL}$ & $<0.03 \mathrm{ng} / \mathrm{mL}$ \\
\hline Chromosome analysis & & $46, X Y$ \\
\hline
\end{tabular}

We next performed nanogold labeling of the patient's testes with a SCC antibody. As shown in Fig. 2H, SCC staining is visible. With higher magnification (Fig. 2I), SCC gold particles were present only in the mitochondria, with negligible nonspecific labeling, confirming that the patient's testes expressed SCC that was not degraded. Because mitochondria are in close proximity to the ER, we hypothesized that the ER structure might be also affected. EM analysis showed that testicular cells were fibrous, and the ER region displayed a loss in ultrastructure. The fibers were present near the mitochondria, suggesting that these are possibly microfilaments. Although the presence of the ACTH receptor in testes remains to be confirmed, using different oligopeptides, the N-terminal fragment of ACTH can stimulate androgen production in a manner similar to adrenal glands (1). Fetal Leydig cells are responsive to both $\mathrm{LH}$ and ACTH; therefore, it is possible that both
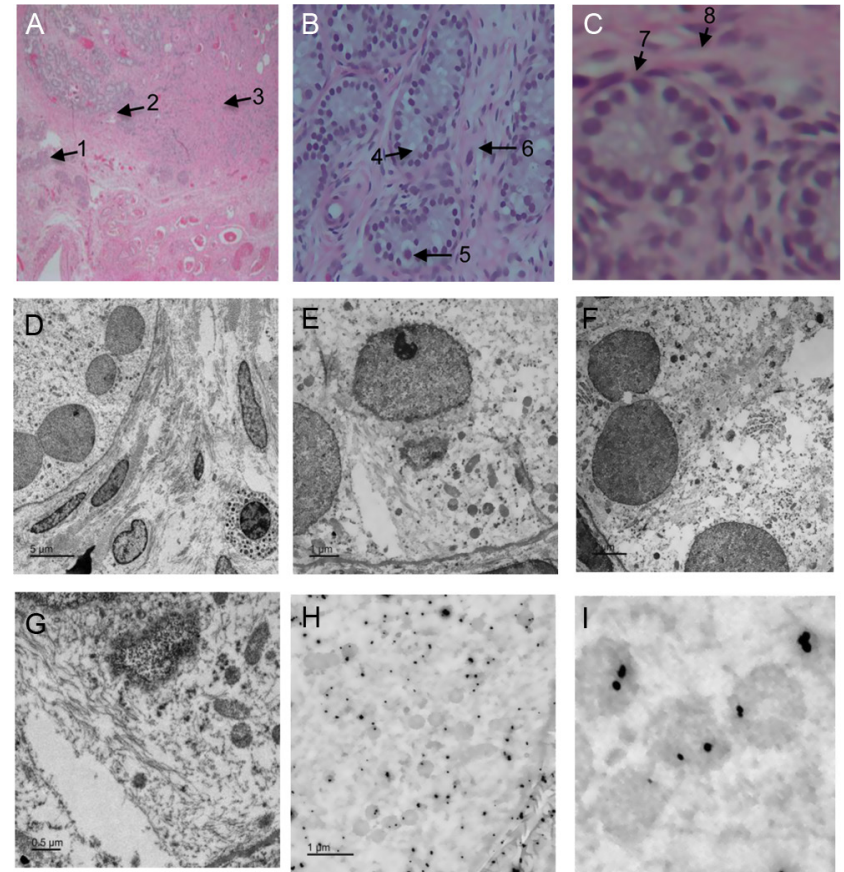

\section{Figure 2}

Histological analysis of the testis of the patient (top) and an unaffected (bottom) baby of similar age. (A) Low-resolution photograph of the complete testicular tissue showing the epididymis (1), stroma (2), and rete testis (3). (B) Shows the location of tubules (4), germ cells (5), and fibrous tissue (6). (C) Stromal fibrous tissue (7) and smaller tubule (8). (D and E) Show the rare location of only two germ cells in the whole testis. The respective bottom panels shown are from an unaffected baby of similar age. (D) Overall EM picture of the patient's testis showing very small sized mitochondria. (E) Staining with an SCC antibody from panel D showed the expression of SCC in the amplified version. Scale, $1.0 \mu \mathrm{m}$. (F, G, H and I) Large-thickness tissue section ( $F$ and $G$ ) and small-thickness section $(\mathrm{H}$ and I), showing the small mitochondria (I) stained with SCC antibody. (G) $5 \mu \mathrm{m}$ and $(\mathrm{H}) 2 \mu \mathrm{m}$ sections show the small mitochondria. 
LH and ACTH may play a role in regulating Leydig cell function (1).

\section{Outcome and follow-up}

The patients testes were removed and currently doing fine.

\section{Discussion}

$\mathrm{CAH}$ is an inherited disorder of the adrenal glands that affects both boys and girls, as the $46 \mathrm{XY}$ male has normal female looking external genitalia. As the adrenal glands are located on the top of kidneys, the electrolytes and carbohydrates of CAH patients are differentially affected depending on the severity of the mutation of the specific participating gene present in the specific pathway (1). However, CAH caused by mutation of the 21-hydroxylase gene is the most common as compared to mutations caused by the other genes, including cytochrome P450scc or 3-beta-hydroxysteroid dehydrogenase or cytochrome P450C17 (1). For example, there are only 20 case reports of SCC mutation causing CAH (7). In our case, the high pigmentation was suggestive of excess ACTH production since it accompanies the production of $\alpha$-melanocytestimulating hormone ( $\alpha$-MSH), from the same precursor, pro-opiomelanocortin (POMC).

In addition to the high pigmentation, the patient had extremely high ACTH levels. However, cortisol levels were undetectable due to a failure of negative feedback on the hypothalamus, resulting in excessive secretion of hypothalamic $\mathrm{CRH}$ and increased ACTH secretion from the anterior pituitary. Continuous secretion of ACTH causes unremitting stimulation of the adrenal cortex, leading to cortical adrenal hyperplasia. The excess ACTH possibly generated a large excess of StAR protein, which may facilitate entry of a large amount of cholesterol into the mitochondria; however, it is unlikely that the small mitochondrial import channel as well as limited SCC could accommodate entry of this large pool of cholesterol into the mitochondria. Since the large number of cholesterol molecules needs a clearing mechanism (14), the cholesterol may remain outside of the mitochondria, resulting in shrinkage of this organelle and increased force generated by the fibrous tissues, which may lead to smaller-sized germ cells.

Another possibility is that the excess ACTH might have created steric hindrance due to the overexpression of proteins, resulting in overcrowding of the mitochondria. To examine the overcrowding pattern, we analyzed the architecture around the ER in the testis of the same patient. As shown in Fig. 3B, this region was overcrowded, which was evident in the enlarged version (Fig. 3C); however, the mitochondria were not close, and the mitochondria associated-ER membrane (MAM) was not visible (5). Further analysis of the cytoplasmic region at a higher magnification ( $5 \mu \mathrm{m}$; Fig. 3D) showed the presence of mitochondria; however, empty spaces were evident in the cytoplasm with no filaments as seen with the enlarged version (Fig. 3E). Further analysis of the microfilaments (Fig. 3F) showed a disorganized architecture, which was evident in the enlarged version (Fig. 3G). In summary, we conclude that an absence of required pregnenolone secretion affected organelle structure.

Mitochondria in the gonads, and, perhaps also in the adrenals, of the patient were overactive and, thus, the OMM and IMM were clipping. Alternatively, the space between the OMM and IMM might be close (i.e., beyond the level of detection for ER) to rapidly transport cholesterol from the cytoplasm to the mitochondria in response to physiological demand. Also, the higher lipid content in the cytoplasm might have induced steric hindrance, pushing the adrenal and gonadal OMM close to the IMM, which might bypass the need for an

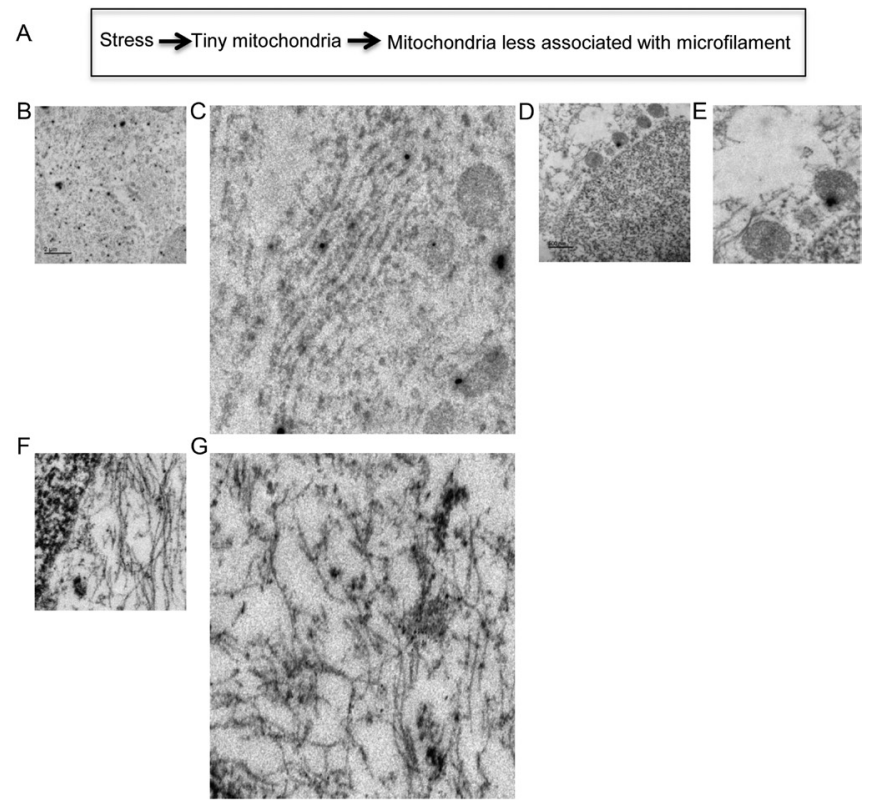

Figure 3

Direct visualization of fiber formation in the testis. (A) Schematic presentation showing the different steps involved in stress-related filament formation. (B and C) Smaller section of the ER region (B), showing that this region of the affected testis is unchanged (C). (D and E) Mitochondria is less associated with the microfilament $(E)$. ( $F$ and $G$ ) Identification of unorganized microfilament $(F)$ with clear presentation in the enlarged panel (G). 
aqueous proteinous channel for cholesterol import. In summary, the absence of pregnenolone secretion altered mitochondrial membrane organization.

\section{Conclusion}

The acute response to stress consists of a relatively stereotyped series of physiological programs to promote survival mediated by the HPA axis, resulting in hypothalamic CRH secretion that stimulates release of ACTH by the pituitary and subsequent adrenal and gonadal glucocorticoid release. Steroid synthesis starts at the mitochondria of adrenals and gonads, and mitochondrial stress may also induce retrograde signaling in mammalian cells (11). Cells can also respond to stress in a way that is specific to individual organelles and propagate to mitochondria. Specifically, the ER stress response or the unfolded protein response (UPR) is activated in response to mild or short-term stress triggers, inducing the expression of a wide range of genes involved in the maintenance of ER function (11). The impaired stress response arises from either primary defects in the adrenal gland or secondary hypothalamic or pituitary defects, resulting in steroidogenesis defects. So, the presence of increased ACTH upregulated expression of the protein (9).

SCC activity requires interaction with the IMM translocase, Tim50, or possibly the TIM23 complex for appropriate folding and activity. Drosophila expressing a Tim50 mutant had growth defects and reduced mitochondrial membrane potential, which is similar to that seen in zebrafish models (11). These studies suggest that Tim50 needs to interact with SCC to be capable of inducing metabolic activity with the other interacting partners, such as ferrodoxin, ferrodoxin reductase and NADPH for six electron pairs. The electron transport system is the microenvironment for metabolic reactions but the global environment requires the formation of the Tim50 complex associated with SCC. Thus, Tim50 mutations inhibited overall interaction with SCC, which prevented pregnenolone synthesis, resulting in ambiguous genitalia (11).

The patient described in the present study with a mutant SCC protein developed enlarged adrenals as well as smaller testes with female appearing external genitalia. Masculinization refers to the process via which the sexually indifferent fetus with testes is transformed into a phenotypic male with internal and external male genitalia; this process cannot occur until testes have differentiated and begun to secrete hormones. Therefore, masculinization follows sexual differentiation but is separate from it. Thus, a genotypic male (XY) fetus can undergo sexual differentiation and form testes but fail to completely masculinize or a genotypic female (XX) fetus without testes can masculinize if exposed to sufficient androgens (15). Because much of the masculinization process involves androgen action and the main source of androgens is the fetal testis, disorders of androgendependent masculinization can provide a 'read-out' of fetal testicular steroidogenic function. Therefore, the type of genital abnormality that is present may provide information as to when the deficiency in androgen action occurred. For the patient described here, androgen was absent due to not only a defect in the SCC gene but also participation of the IMM translocase complex. The testes had fibrous tissue as determined by histological analysis (Fig. 2B), and the mitochondrial size was extremely small (Fig. $3 \mathrm{H}$ ), suggesting that steric hindrance affected organelle architecture. In summary, proteins inside the cells were misfolded due to the absence of steroids, which affected the development of organelle architecture as well as activity.

\section{Declaration of interest}

Ethics and consent to participate: The sample was collected by Dr Alan Rice MD, as laid by the Institutional Review Board. Availability of data and material: Original data will be available on written request to $\mathrm{Dr}$ Bose. The other authors have nothing to disclose.

\section{Funding}

The work was supported by Navicent Grant Foundation and a Seed Grant from the Mercer U School of Medicine (all to H S B).

\section{Patient consent}

Written, informed consent has been obtained from the patient for the publication of this article.

\section{Author contribution statement}

H S B conceptualized the project, designed experiments, analyzed the data and wrote the manuscript; F G, A M R, E W P and B M performed the experiments. A complete manuscript was available to all of the authors prior to initial submission.

\section{References}

1 Miller WL \& Auchus RJ. The molecular biology, biochemistry, and physiology of human steroidogenesis and its disorders. Endocrine Reviews 201132 81-151. (https://doi.org/10.1210/er.2010-0013)

2 Ferguson JJ. Protein synthesis and adrenocorticotropin responsiveness. Journal of Biological Chemistry 1963238 2754-2759. 
3 Garren LD, Ney RL \& Davis WW. Studies on the role of protein synthesis in the regulation of corticosterone production by ACTH in vivo. PNAS 196553 1443-1450. (https://doi.org/10.1073/ pnas.53.6.1443)

4 Davia WW \& Garren LD. On the mechanism of action of adrenocorticotropic hormone. The inhibitory site of cycloheximide in the pathway of steroid biosynthesis. Journal of Biological Chemistry 1968243 5153-5157.

5 Prasad M, Pawlak KJ, Burak WE, Perry EE, Marshall B, Whittal RM \& Bose HS. Mitochondrial metabolic regulation by GRP78. Science Advances 20173 e1602038. (https://doi.org/10.1126/sciadv.1602038)

6 Kaur J, Rice AM, O'Conner E, Piya A, Buckler B \& Bose HS. Novel SCC mutation in a patient of Mexican descent with sex reversal, salt-losing crisis and adrenal failure. Endocrinology, Diabetes and Metabolism Case Reports 20162016 ID: 16-0059. (https://doi.org/10.1530/EDM-16-0059)

7 Nimkarn S, Cerame BI, Wei JQ, Dumic M, Zunec R, Brkljacic L, Skrabić V, New MI \& Wilson RC. Congenital adrenal hyperplasia (21-hydroxylase deficiency) without demonstrable genetic mutations. Journal of Clinical Endocrinology and Metabolism 199984 378-381. (https://doi.org/10.1210/jcem.84.1.5554)

8 Koopman P, Münsterberg A, Capel B, Vivian N \& Lovell-Badge R. Expression of a candidate sex-determining gene during mouse testis differentiation. Nature 1990348 450-452. (https://doi. org/10.1038/348450a0)

9 Prasad M, Walker AN, Kaur J, Thomas JL, Powell SA, Pandey AV, Whittal RM, Burak WE, Petruzzelli G \& Bose HS. Endoplasmic reticulum stress enhances mitochondrial metabolic activity in mammalian adrenals and gonads. Molecular and Cellular Biology 2016 36 3058-3074. (https://doi.org/10.1128/MCB.00411-16)

10 Rajapaksha M, Kaur J, Prasad M, Pawlak KJ, Marshall B, Perry EW, Whittal RM \& Bose HS. An outer mitochondrial translocase, Tom22, is crucial for inner mitochondrial steroidogenic regulation in adrenal and gonadal tissues. Molecular and Cellular Biology 201636 1032-1047. (https://doi.org/10.1128/MCB.01107-15)

11 Evetts AM, Shkrum MJ \& Tugaleva E. A new reference source for postmortem body measurements and organ weights in neonates and infants: A statistical analysis based on sudden death classification (Part 2). American Journal of Forensic Medicine and Pathology 201839 285-303. (https://doi.org/10.1097/PAF.0000000000000401)

12 Miller WL \& Bose HS. Early steps in steroidogenesis: intracellular cholesterol trafficking. Journal of Lipid Research 201152 2111-2135. (https://doi.org/10.1194/jlr.R016675)

13 Rajapaksha M, Kaur J, Bose M, Whittal RM \& Bose HS. Cholesterolmediated conformational changes in the steroidogenic acute regulatory protein are essential for steroidogenesis. Biochemistry 2013 52 7242-7253. (https://doi.org/10.1021/bi401125v)

14 Bose HS, Gebrail F, Marshall B, Perry EW \& Whittal RW. Inner mitochondrial translocase Tim 50 is central in adrenal and testicular steroid synthesis. Molecular and Cellular Biology 201939 doi: doi:10.1128/MCB.00484-00418.

15 Hughes IA. Miniriview: sex differentiation. Endocrinology 2001142 3281-3287. (https://doi.org/10.1210/endo.142.8.8406)

Received in final form 4 April 2019

Accepted 11 April 2019 